\title{
An Assessment of the Drought Index as Impact of Climate Change Using MockWyn-UB Model
}

\author{
I WayanSutapa $^{1, \mathrm{a}}$, Saiful Darman ${ }^{2, \mathrm{~b}}$, Djayani Nurdin ${ }^{3, \mathrm{c}}$, Fathurrahman ${ }^{4, \mathrm{~d}}$ \\ ${ }^{1}$ Department of Civil Engineering, Faculty of Engineering, University of Tadulako, Palu \\ ${ }^{2}$ Department of Agriculture, Faculty of Agriculture, University of Tadulako, Palu \\ ${ }^{3}$ Department Economy, Faculty of Economy, University of Tadulako, Palu \\ ${ }^{4}$ Department of Agriculture, Faculty of Agriculture, University of Tadulako, Palu \\ E-mail: ${ }^{\mathrm{a} w s u t a p a @ y a h o o . c o m ~(c o r r e s p o n d i n g ~ a u t h o r), ~}{ }^{\mathrm{b}}$ saifuldarman@yahoo.co.id, \\ cdjayani.nurdin@gmail.com, ${ }^{\mathrm{d}}$ fathurrahmanshahib@ymail.com
}

\begin{abstract}
This study intended to investigate the effect of climate change on the drought index in Bangga Watershed. The investigation was carried out by comparing water deficit and potential evapotranspiration. Water deficit was analyzed due to water balance by using MockWyn-UB model which was as the development of FJ Mock model. The scenario on the effect of climate change was based on the results of detection and projection of climate change by using a Make sens method which there was yearly rainfall decreasing of $20 \%$ and temperature increase of about $1^{0} \mathrm{C}$ during the observation period of historical data. The results showed that the average of drought level with the small scale was occurred on February until August, the medium scale between September until December, and big scale on December. After climate change, there was increasing of drought index about $15 \%$ until $60 \%$ which drought level on the medium scale has occurred on February until September and drought level on the big scale has happened on January, October until December.
\end{abstract}

Keywords:Bangga Watershed, climate change, drought index, MockWyn-UB model

\section{INTRODUCTION}

Water is as the most generous substation in the earth surface, the main component for all of the living creature, and as the main strength which constantly forms the earth surface. Water is also as the determining factor in regulating the climate in earth surface for the demand of human life [1]. The effect of climate change is marked by the happening of the season moving that causes long dry season so there is occurred the drought which is influenced agricultural sector [2-4]. The role of climate change affects the increase in rainfall, which causes an increased risk of landslides in the hills [5]

To more optimize the management of water resources, one of them is necessary to be carried out the analysis of water balance [6, 7]. Generally, water balance presents the relation between inflow and outflow in a region at the certain time. The water balance is very necessary for evaluating the availability of rainfall in a region especially for knowing when and how much the water surplus and deficit which is happened in the location of study. By the result of water balance analysis, it can be carried out indirectly to the components of water balance which is not known the volume based on the known component like water deficit or surplus on the certain month in this region.

The aim of this study is to investigate the effect of climate change on the drought index in Bangga watershed. An investigation was carried out by comparing water deficit and potential evapotranspiration. Water deficit was analyzed by using the model of MockWyn-UB [8]. The scenario on the influence of climate change based on the result of detection and projection of climate change by using Make sens method which during the observation period of historical data there was the decreasing of yearly rainfall of $20 \%$ and the increasing of temperature of about $1^{\circ} \mathrm{C}$ [8]. This study was very useful for knowing water deficit as well as surplus from drought index [9] so that was used as the consideration in water management in a region. Drought index was classified into three scales such as small-scale (to be not), medium scale, and big scale with the value range in gradually of 0 to $16.7 ; 15.7$ to 33.3 ; and 33.3 to 100 [10]. 


\section{METHODOLOGY}

The research location is located in the Bangga River Basin, Central Sulawesi, Indonesia, where its watershed area is $65.90 \mathrm{~km}^{2}$. For more details are presented in Figure 1: $[8,11]$.

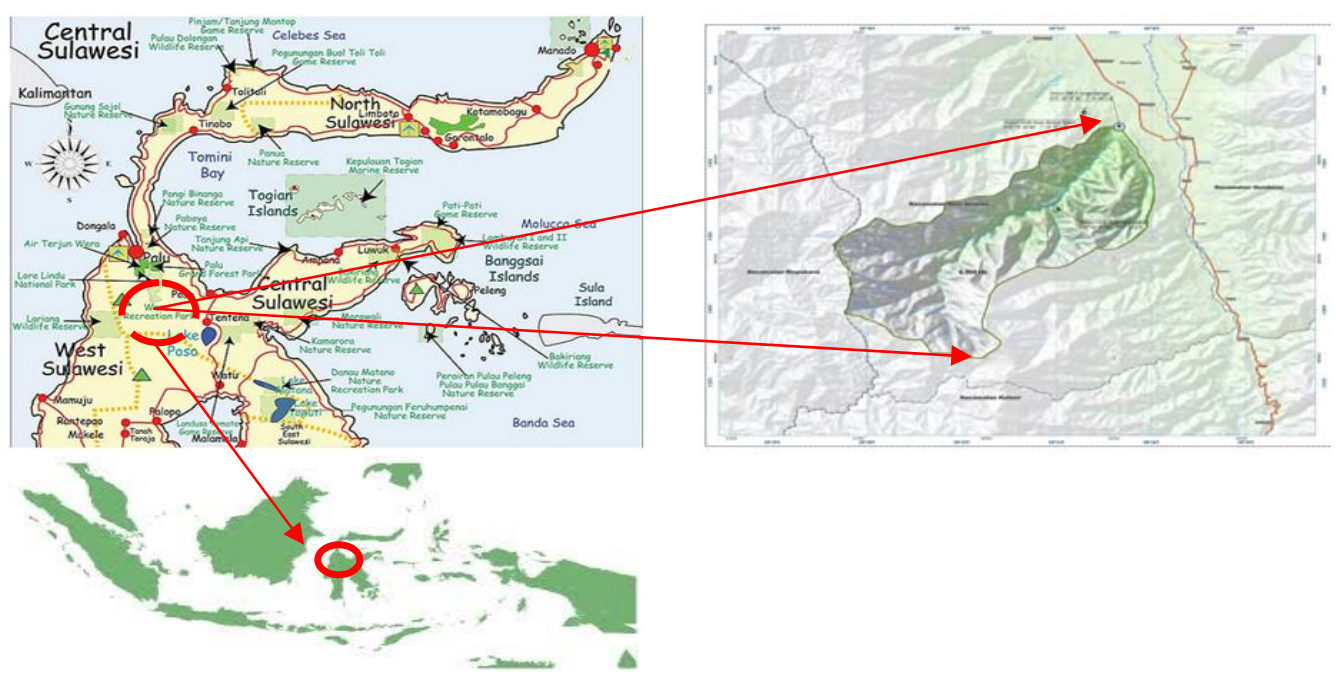

Fig. 1. Location of the research

The data used in this research are secondary and primary data. Primary data obtained directly from the research location in the form of soil characteristics, while secondary data obtained from the Regional Office of Sulawesi River Region III in Palu, Central Sulawesi, Indonesia. Secondary data required in the form of daily rainfall data and climatology data. The location of the nearest rain station is Upper Bangga Station and Bottom Bangga Station, while climatology data using Bora Climatology Station. The earth map for the research location was obtained from the Office of the National Survey and Mapping Coordination Agency in Jakarta, Indonesia. Maps available on a 1: 50,000 scale.[8, 11].

To investigate of climate change influence to the drought index, it was necessary the method which the steps were as follow: 1) analysis of rainfall; 2) analysis of potential evapotranspiration; 3) analysis of water balance; 4) analysis of drought index.

Some of the analysis done on the MockWyn-UB model are[8, 11-24]:

1). Detection and projection of climate change; 2).Rainfall-based on the area of land use $\left(\mathrm{T}_{\mathrm{PN}}\right)$; 3).Potential evapotranspiration (ETo); 4). Actual evapotranspiration (ETa); 5).Difference between $\mathrm{T}_{\mathrm{PN}}$ and ETo per month; 6). Accumulated Potential Water Loss (APWL); 7). Soil Moisture (SM); 8). Changes in soil moisture per month $(\Delta \mathrm{SM})$; 9).Water surplus (WS); 10). Groundwater storage(Vn); 11). Storm Runoff (SR); 12). River Flow discharge $(\mathrm{Q})$ in the form of base flow and direct runoff.

While to analyze the Drought Index used additional formulas as follows:

Water Deficit (WD) = ETo - Eta

Drought index (Ia) $\quad=$ WD $/$ ETo

\section{RESULTS AND DISCUSSION}

Table 1 presented the analysis of drought index in 1995 (for example), and Table 2 presented the result of drought index from 1995 to 2011. Figure 2, 3, and 4 presented the correlation between rainfall (R) and evapotranspiration (ETo) with drought index (Ia) each from 1995 to 2000, 2001 to 2006, and 2007 to 2011. Figure 5 presented the correlation between R and Eto with Ia in monthly average from 1995 to 2011. 
TABLE I. Analysis of Drought Index in 1995 (for example)

\begin{tabular}{|c|c|c|c|c|c|c|c|c|c|c|c|c|c|}
\hline No. & Item & $\operatorname{Jan}$ & Feb & Mar & Apr & May & Jun & Jul & Aug & Sep & Oct & Nop & Dec \\
\hline \multicolumn{14}{|c|}{ I Vegetation } \\
\hline 1 LHT & & 56.85 & $\mathrm{Km}^{2}$ & & & & & & & & & & \\
\hline 2 LKC & & 8.25 & $\mathrm{Km}^{3}$ & $\stackrel{\square}{>}$ & & & & & & & & & \\
\hline 3 LLT & & 0.8 & $\mathrm{Km}^{4}$ & $\frac{1}{5}$ & & & & & & & & & \\
\hline 4 LDAS & & 65.9 & $\mathrm{Km}^{5}$ & & & & & & & & & & \\
\hline
\end{tabular}

\section{RAINFALL \& EVAPO.}

$5 P$

6 PHT

7 PKC

8 PLT

9 PNHT

10 PNKC

11 PNLT

12 TPN

13 ETO

$14 \mathrm{ETa}$ $\begin{array}{llllllllllll}155.00 & 248.60 & 265.55 & 97.85 & 135.35 & 182.95 & 169.20 & 232.15 & 287.35 & 205.05 & 191.80 & 28.40\end{array}$

$\begin{array}{llllllllllll}133.71 & 214.46 & 229.08 & 84.41 & 116.76 & 157.83 & 145.96 & 200.27 & 247.89 & 176.89 & 165.46 & 24.50\end{array}$

$\begin{array}{llllllllllll}19.40 & 31.12 & 33.24 & 12.25 & 16.94 & 22.90 & 21.18 & 29.06 & 35.97 & 25.67 & 24.01 & 3.56\end{array}$

$\begin{array}{llllllllllll}1.88 & 3.02 & 3.22 & 1.19 & 1.64 & 2.22 & 2.05 & 2.82 & 3.49 & 2.49 & 2.33 & 0.34\end{array}$

$\begin{array}{llllllllllll}118.56 & 190.10 & 203.05 & 74.88 & 103.54 & 139.92 & 129.41 & 177.53 & 219.72 & 156.81 & 146.69 & 21.79\end{array}$

$\begin{array}{llllllllllll}18.28 & 29.12 & 31.08 & 11.66 & 16.01 & 21.52 & 19.93 & 27.22 & 33.61 & 24.08 & 22.54 & 3.62\end{array}$

$\begin{array}{llllllllllll}1.88 & 3.02 & 3.22 & 1.19 & 1.64 & 2.22 & 2.05 & 2.82 & 3.49 & 2.49 & 2.33 & 0.34\end{array}$

$\begin{array}{llllllllllll}138.72 & 222.24 & 237.36 & 87.73 & 121.19 & 163.66 & 151.39 & 207.56 & 256.81 & 183.38 & 171.56 & 25.76\end{array}$

$\begin{array}{llllllllllll}124.31 & 109.20 & 124.62 & 120.30 & 123.38 & 105.30 & 108.50 & 111.91 & 123.00 & 126.79 & 113.70 & 111.29\end{array}$

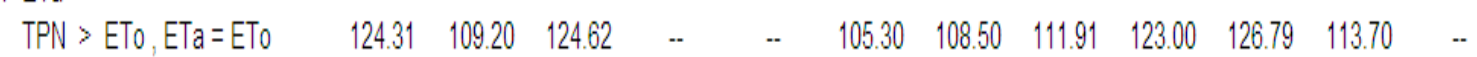
\begin{tabular}{lllllllllllllll}
$\mathrm{TPN}$ & $\mathrm{EET}, \mathrm{ETa}=\mathrm{TPN}+\triangle \mathrm{SSl}$ &. & $\ldots$ & $\ldots$ & $\ldots$ & 118.84 & 123.18 & $\ldots$ & $\ldots$ & $\ldots$ & $\ldots$ & $\ldots$ & $\ldots$ & 101.68 \\
\hline
\end{tabular}

\section{WATER BALANCE}

\begin{tabular}{|c|c|c|c|c|c|c|c|c|c|c|c|c|}
\hline $15 \mathrm{~S}=\mathrm{TPN}-\mathrm{ETO}$ & 14.41 & 113.04 & 112.74 & -32.57 & -2.19 & 58.36 & 42.89 & 95.65 & 133.81 & 56.59 & 57.86 & .85 .53 \\
\hline 16 Potensial water losses & 0.00 & 0.00 & 0.00 & -32.57 & -2.19 & 0.00 & 0.00 & 0.00 & 0.00 & 0.00 & 0.00 & -85.53 \\
\hline 17 APWL & 0.00 & 0.00 & 0.00 & -32.57 & -34.76 & 0.00 & 0.00 & 0.00 & 0.00 & 0.00 & 0.00 & -85.53 \\
\hline $18 S M=S M C \cdot e^{\wedge} \cdot(A P W L / S M C)$ & 351.55 & 351.55 & 351.55 & 320.44 & 318.45 & 351.55 & 351.55 & 351.55 & 351.55 & 351.55 & 351.55 & 275.63 \\
\hline $19 \Delta S M$ & 0.00 & 0.00 & 0.00 & -31.11 & -1.99 & 33.10 & 0.00 & 0.00 & 0.00 & 0.00 & 0.00 & 5.92 \\
\hline $20 \mathrm{WD}=\mathrm{ET} 0 \cdot \mathrm{ETa}$ & 0 & 0 & 0 & 1.46 & 0.20 & 0 & 0 & 0 & 0 & 0 & 0 & 961 \\
\hline 21 WS & 14.41 & 113.04 & 112.74 & 0.00 & 0.00 & 58.36 & 42.89 & 95.65 & 133.81 & 56.59 & 57.86 & 000 \\
\hline $22 \mathrm{la}$ & 0.00 & 0.00 & 0.00 & 1.22 & 0.16 & 0.00 & 0.00 & 0.00 & 0.00 & 0.00 & 0.00 & 8.63 \\
\hline
\end{tabular}


TABLE II. Recapitulation of Drought Index Analysis in 1995 to 2011

\begin{tabular}{|c|c|c|c|c|c|c|c|c|c|c|c|c|c|}
\hline Item & Year & Jan & Feb & Mar & Apr & May & Jun & Jul & Aug & Sep & Oct & Nov & Dec \\
\hline Monthly rainfall (R) & & 186.00 & 298.32 & 318.66 & 117.42 & 162.42 & 219.54 & 203.04 & 278.58 & 344.82 & 246.06 & 230.16 & 34.08 \\
\hline ETo & & 124.31 & 109.20 & 124.62 & 120.30 & 123.38 & 105.30 & 108.50 & 111.91 & 123.00 & 126.79 & 113.70 & 111.29 \\
\hline la-1 & 1995 & 0.00 & 0.00 & 0.00 & 1.22 & 0.16 & 0.00 & 0.00 & 0.00 & 0.00 & 0.00 & 0.00 & 8.63 \\
\hline $\mid a-2$ & & 12.83 & 0.00 & 0.00 & 3.09 & 4.08 & 0.00 & 0.00 & 0.00 & 0.00 & 0.00 & 0.00 & 9.97 \\
\hline Monthly rainfall (R) & & 135.24 & 254.40 & 118.86 & 179.28 & 186.84 & 195.90 & 108.90 & 214.32 & 180.66 & 134.22 & 96.90 & 49.92 \\
\hline ETo & & 144.46 & 136.64 & 164.61 & 142.20 & 144.77 & 120.30 & 124.62 & 144.46 & 145.20 & 140.74 & 134.70 & 132.99 \\
\hline la-1 & 1996 & 30.10 & 0.00 & 4.63 & 1.21 & 0.82 & 0.00 & 2.05 & 0.00 & 0.11 & 2.39 & 9.57 & 26.22 \\
\hline la-2 & & 45.54 & 0.00 & 7.36 & 7.57 & 8.94 & 2.13 & 22.20 & 7.13 & 15.34 & 27.40 & 39.89 & 59.22 \\
\hline Monthly rainfall (R) & & 44.46 & 113.94 & 120.72 & 204.60 & 49.38 & 72.54 & 198.90 & 7.20 & 16.38 & 27.66 & 319.80 & 60.96 \\
\hline ETo & & 115.94 & 95.20 & 123.07 & 135.60 & 134.23 & 139.80 & 125.55 & 162.75 & 151.80 & 148.80 & 135.00 & 129.27 \\
\hline la-1 & 1997 & 71.12 & 2.33 & 7.11 & 0.00 & 9.13 & 19.95 & 0.00 & 18.66 & 43.25 & 54.88 & 0.00 & 7.10 \\
\hline la-2 & & 77.37 & 7.77 & 14.65 & 4.98 & 39.38 & 43.23 & 5.10 & 72.73 & 78.12 & 79.13 & 0.00 & 9.00 \\
\hline Monthly rainfall $(\mathrm{R})$ & & 11.94 & 17.76 & 83.22 & 197.34 & 115.98 & 163.02 & 316.32 & 103.86 & 167.46 & 85.32 & 49.26 & 26.70 \\
\hline ETo & & 151.59 & 143.64 & 156.55 & 130.50 & 125.86 & 112.20 & 115.63 & 119.35 & 124.80 & 130.82 & 106.80 & 114.39 \\
\hline la-1 & 1998 & 93.87 & 40.04 & 35.86 & 0.00 & 1.67 & 0.00 & 0.00 & 1.99 & 0.00 & 4.58 & 16.34 & 33.35 \\
\hline $\mid a-2$ & & 95.16 & 42.16 & 42.56 & 8.15 & 32.97 & 11.34 & 0.00 & 3.99 & 4.13 & 18.93 & 32.70 & 49.36 \\
\hline Monthly rainfall $(\mathrm{R})$ & & 5.40 & 36.78 & 64.92 & 162.54 & 123.36 & 24.72 & 71.46 & 113.16 & 112.98 & 176.34 & 43.74 & 16.74 \\
\hline ETo & & 107.88 & 102.20 & 113.15 & 117.60 & 111.29 & 107.40 & 111.60 & 132.06 & 127.20 & 128.03 & 120.00 & 127.41 \\
\hline la-1 & 1999 & 95.89 & 23.92 & 25.59 & 0.00 & 0.46 & 13.44 & 16.72 & 14.97 & 16.37 & 0.00 & 8.28 & 30.01 \\
\hline la-2 & & 96.70 & 26.87 & 31.45 & 10.62 & 20.53 & 56.60 & 45.46 & 38.88 & 39.18 & 16.48 & 67.73 & 82.75 \\
\hline Monthly rainfall (R) & & 249.18 & 27.12 & 31.98 & 122.88 & 133.32 & 206.52 & 86.82 & 80.52 & 106.08 & 82.62 & 120.24 & 33.00 \\
\hline ETo & & 113.77 & 108.92 & 127.41 & 125.10 & 132.06 & 99.30 & 115.63 & 119.35 & 132.00 & 110.05 & 118.50 & 106.02 \\
\hline la-1 & 2000 & 0.00 & 9.39 & 26.41 & 11.89 & 12.21 & 0.00 & 3.01 & 10.05 & 12.79 & 18.01 & 11.43 & 41.89 \\
\hline la-2 & & 0.00 & 10.56 & 29.44 & 20.81 & 22.12 & 0.00 & 5.00 & 15.39 & 20.90 & 27.97 & 23.41 & 52.93 \\
\hline Monthly rainfall $(\mathrm{R})$ & & 131.40 & 61.32 & 71.52 & 175.92 & 158.88 & 135.72 & 28.56 & 18.66 & 108.72 & 78.96 & 25.38 & 57.00 \\
\hline ETo & & 114.08 & 93.24 & 122.14 & 112.80 & 115.63 & 106.50 & 120.28 & 137.33 & 130.50 & 138.57 & 115.80 & 118.11 \\
\hline la-1 & 2001 & 13.99 & 5.36 & 13.52 & 0.00 & 0.00 & 0.04 & 11.54 & 33.32 & 19.26 & 33.93 & 56.83 & 47.89 \\
\hline $\mid a-2$ & & 32.76 & 10.63 & 21.15 & 3.68 & 8.72 & 12.21 & 48.51 & 63.17 & 39.22 & 54.21 & 74.67 & 63.99 \\
\hline Monthly rainfall $(\mathrm{R})$ & & 84.12 & 13.26 & 2.04 & 0.00 & 67.74 & 243.30 & 106.62 & 29.88 & 127.62 & 66.24 & 222.72 & 81.18 \\
\hline ETo & & 124.93 & 110.88 & 120.59 & 131.10 & 123.38 & 98.70 & 134.85 & 143.53 & 133.20 & 145.08 & 129.30 & 135.47 \\
\hline la-1 & 2002 & 49.60 & 24.58 & 45.80 & 62.27 & 42.36 & 0.00 & 3.05 & 23.30 & 12.10 & 34.42 & 0.00 & 5.46 \\
\hline la-2 & & 60.59 & 28.43 & 49.09 & 64.85 & 50.53 & 0.00 & 5.35 & 28.33 & 21.39 & 43.51 & 0.00 & 7.66 \\
\hline Monthly rainfall (R) & & 44.40 & 73.68 & 91.56 & 179.76 & 125.76 & 43.44 & 72.54 & 92.28 & 33.24 & 146.88 & 128.52 & 33.96 \\
\hline ETo & & 117.80 & 106.40 & 115.32 & 120.60 & 126.48 & 127.80 & 112.84 & 122.76 & 114.60 & 127.41 & 126.60 & 100.75 \\
\hline la-1 & 2003 & 71.62 & 12.89 & 14.75 & 0.00 & 1.16 & 14.98 & 18.62 & 19.86 & 43.22 & 8.62 & 15.55 & 51.60 \\
\hline a-2 & & 77.75 & 17.84 & 22.31 & 6.20 & 22.26 & 49.86 & 44.11 & 42.68 & 67.20 & 27.70 & 35.34 & 71.15 \\
\hline Monthly rainfall (R) & & 106.14 & 13.92 & 49.14 & 142.14 & 198.48 & 61.02 & 120.36 & 1.02 & 90.18 & 20.70 & 46.62 & 16.92 \\
\hline ETo & & 125.55 & 104.44 & 124.00 & 119.70 & 124.93 & 117.30 & 115.32 & 138.57 & 131.10 & 144.77 & 133.20 & 133.61 \\
\hline la-1 & 2004 & 36.80 & 20.65 & 28.43 & 5.51 & 0.00 & 5.80 & 4.69 & 36.85 & 25.74 & 57.13 & 54.58 & 73.11 \\
\hline a-2 & & 50.60 & 25.24 & 34.56 & 16.85 & 4.44 & 43.80 & 27.15 & 75.78 & 49.50 & 79.82 & 72.32 & 86.43 \\
\hline Monthly rainfall $(\mathrm{R})$ & & 14.46 & 26.52 & 121.86 & 108.90 & 139.26 & 50.28 & 76.50 & 97.02 & 138.72 & 147.18 & 62.94 & 54.12 \\
\hline ETo & & 124.93 & 113.12 & 144.77 & 122.40 & 123.07 & 114.30 & 115.01 & 137.02 & 118.50 & 132.99 & 116.10 & 106.33 \\
\hline la-1 & 2005 & 91.06 & 29.94 & 18.00 & 18.43 & 9.11 & 42.76 & 35.22 & 35.25 & 9.78 & 13.71 & 48.29 & 52.27 \\
\hline la-2 & & 92.95 & 32.64 & 26.25 & 28.89 & 22.32 & 52.92 & 47.17 & 47.87 & 26.85 & 30.54 & 60.40 & 63.67 \\
\hline Monthly rainfall (R) & & 56.88 & 82.80 & 10.26 & 168.30 & 111.78 & 67.08 & 116.64 & 79.62 & 102.66 & 10.92 & 117.48 & 86.04 \\
\hline ETo & & 118.73 & 114.80 & 128.96 & 120.60 & 132.37 & 108.60 & 128.03 & 136.09 & 131.40 & 139.19 & 128.40 & 125.86 \\
\hline la-1 & 2006 & 64.02 & 11.59 & 38.76 & 0.00 & 2.45 & 10.63 & 9.71 & 23.04 & 21.25 & 58.43 & 22.33 & 36.39 \\
\hline a-2 & & 71.81 & 16.78 & 43.65 & 10.53 & 31.31 & 43.77 & 34.62 & 51.99 & 45.36 & 83.41 & 42.29 & 55.32 \\
\hline Monthly rainfall (R) & & 89.10 & 199.86 & 137.40 & 183.48 & 287.94 & 124.14 & 51.24 & 289.80 & 94.92 & 138.66 & 107.70 & 132.96 \\
\hline ETo & & 111.60 & 120.40 & 131.13 & 120.30 & 137.33 & 96.30 & 109.12 & 130.20 & 129.00 & 125.55 & 120.90 & 119.97 \\
\hline la-1 & 2007 & 40.26 & 0.00 & 0.86 & 0.00 & 0.00 & 0.02 & 6.68 & 0.00 & 3.51 & 3.12 & 8.28 & 5.35 \\
\hline la-2 & & 53.30 & 0.52 & 8.81 & 3.31 & 0.00 & 0.82 & 11.99 & 0.00 & 5.67 & 8.63 & 16.61 & 15.36 \\
\hline Monthly rainfall $(\mathrm{R})$ & & 51.00 & 29.04 & 165.84 & 187.98 & 145.08 & 173.04 & 265.98 & 66.18 & 0.00 & 0.00 & 136.44 & 29.82 \\
\hline ETo & & 130.51 & 108.08 & 127.10 & 118.20 & 103.54 & 125.40 & 124.31 & 142.60 & 137.10 & 139.81 & 131.70 & 131.44 \\
\hline la-1 & 2008 & 70.62 & 25.29 & 1.06 & 0.00 & 0.00 & 0.00 & 0.00 & 7.91 & 36.29 & 56.87 & 15.05 & 59.91 \\
\hline la-2 & & 76.93 & 28.86 & 10.70 & 3.45 & 9.00 & 10.36 & 0.00 & 9.96 & 38.90 & 59.03 & 27.33 & 65.56 \\
\hline Monthly rainfall (R) & & 65.04 & 0.00 & 121.92 & 124.38 & 93.24 & 69.84 & 89.16 & 129.12 & 53.28 & 113.10 & 105.78 & 157.32 \\
\hline ETo & & 115.94 & 94.08 & 119.04 & 120.90 & 122.14 & 115.80 & 108.19 & 134.23 & 148.50 & 126.48 & 123.90 & 129.58 \\
\hline la-1 & 2009 & 57.92 & 27.36 & 9.20 & 10.15 & 21.38 & 31.48 & 24.30 & 18.95 & 53.57 & 26.06 & 29.32 & 7.76 \\
\hline a-2 & & 67.06 & 30.08 & 17.39 & 20.35 & 32.41 & 42.83 & 37.38 & 33.44 & 64.43 & 40.91 & 44.17 & 26.18 \\
\hline Monthly rainfall (R) & & 116.46 & 98.88 & 47.04 & 0.00 & 0.00 & 327.24 & 289.56 & 316.68 & 119.94 & 68.28 & 58.44 & 94.50 \\
\hline ETo & & 115.32 & 124.04 & 131.75 & 115.80 & 124.93 & 113.10 & 109.74 & 113.15 & 131.70 & 129.58 & 132.60 & 109.12 \\
\hline la-1 & 2010 & 24.54 & 5.66 & 21.95 & 47.97 & 62.89 & 0.00 & 0.00 & 0.00 & 1.84 & 12.39 & 24.94 & 16.82 \\
\hline |a-2 & & 41.30 & 11.16 & 30.14 & 54.87 & 68.08 & 0.00 & 0.00 & 0.00 & 3.97 & 18.32 & 32.58 & 28.05 \\
\hline Monthly rainfall $(R)$ & & 87.18 & 78.78 & 89.82 & 77.04 & 53.52 & 58.02 & 92.94 & 237.36 & 147.18 & 55.68 & 251.40 & 109.26 \\
\hline ETo & & 122.45 & 96.60 & 115.32 & 111.90 & 122.45 & 110.10 & 104.47 & 127.41 & 117.60 & 134.85 & 121.80 & 112.84 \\
\hline la-1 & 2011 & 46.72 & 7.55 & 11.95 & 18.54 & 32.98 & 35.56 & 21.54 & 0.00 & 0.07 & 9.69 & 0.00 & 1.19 \\
\hline la-2 & & 58.36 & 12.82 & 19.34 & 29.17 & 43.20 & 45.67 & 34.53 & 0.00 & 1.41 & 16.63 & 0.00 & 2.93 \\
\hline Average R & & 86.96 & 83.90 & 96.87 & 137.17 & 126.65 & 131.49 & 135.03 & 126.78 & 114.40 & 94.05 & 124.91 & 63.20 \\
\hline Average ETo & & 122.34 & 110.70 & 128.80 & 122.68 & 125.17 & 112.84 & 116.69 & 132.52 & 131.01 & 133.50 & 124.06 & 120.26 \\
\hline Average la-1 & & 50.48 & 14.50 & 17.88 & 10.42 & 11.58 & 10.27 & 9.24 & 14.36 & 17.60 & 23.19 & 18.87 & 29.70 \\
\hline Average la-2 & & 59.47 & 17.79 & 24.05 & 17.49 & 24.72 & 24.44 & 21.68 & 28.90 & 30.68 & 37.21 & 33.50 & 44.09 \\
\hline Increase la-2 to la- 1 & & $15.12 \%$ & $18.46 \%$ & $25.67 \%$ & $40.41 \%$ & $53.18 \%$ & $57.97 \%$ & $57.37 \%$ & $50.31 \%$ & $42.65 \%$ & $37.68 \%$ & $43.67 \%$ & $32.63 \%$ \\
\hline
\end{tabular}




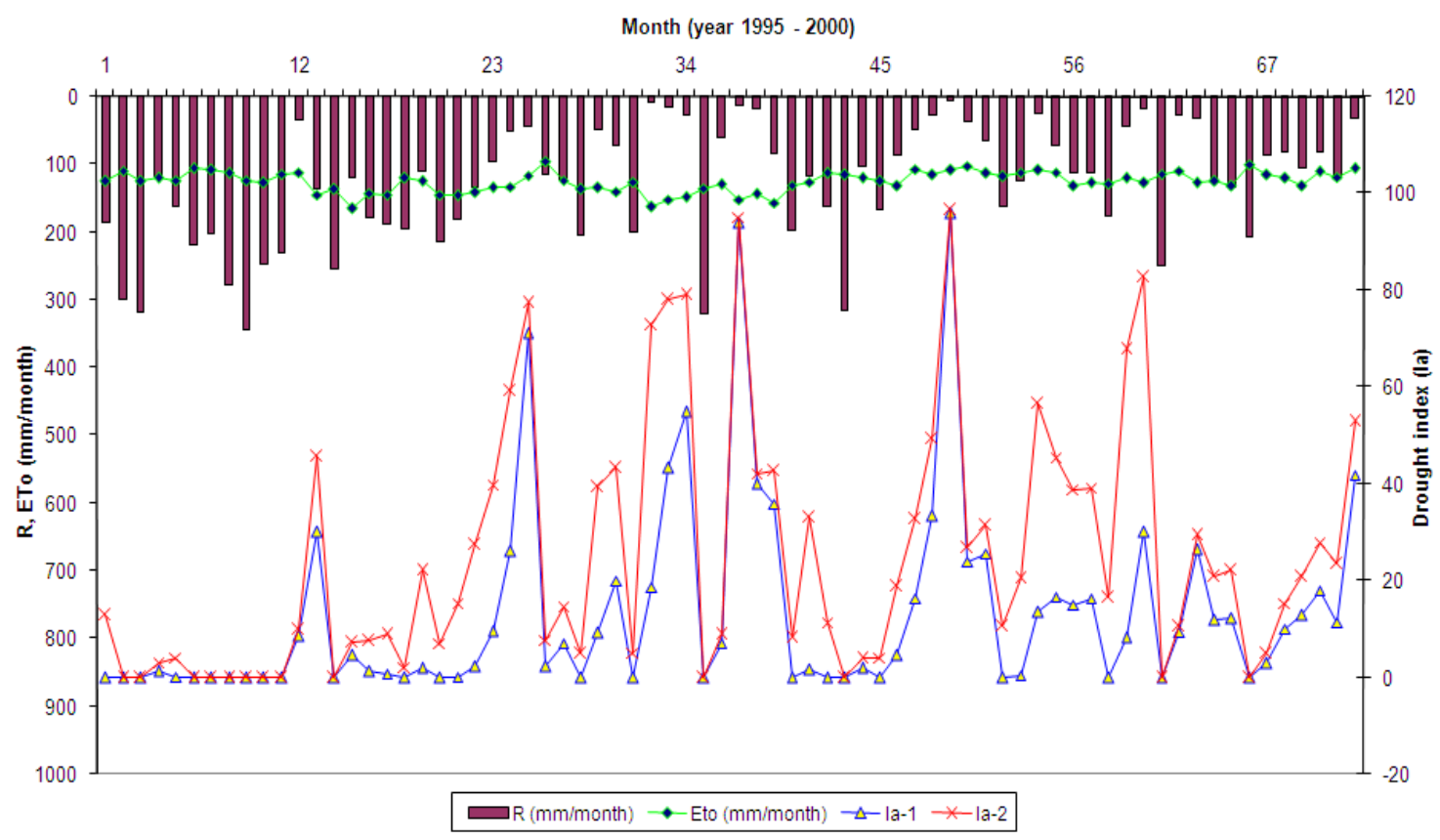

Fig. 2. Correlation between R and ETo with Ia from 1995 to 2000

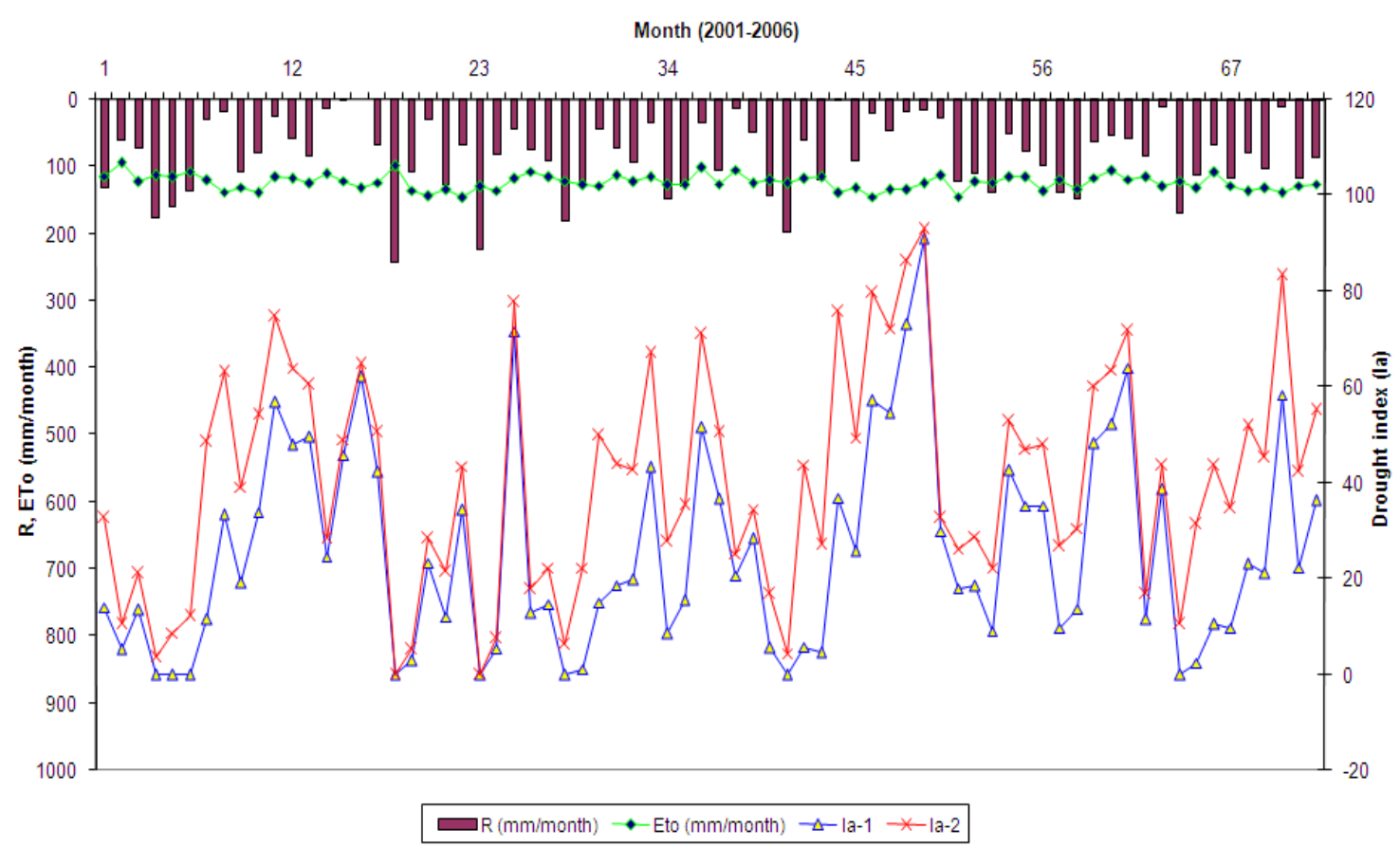

Fig. 3. Correlation between R and ETo with Ia from 2001 to 2006 


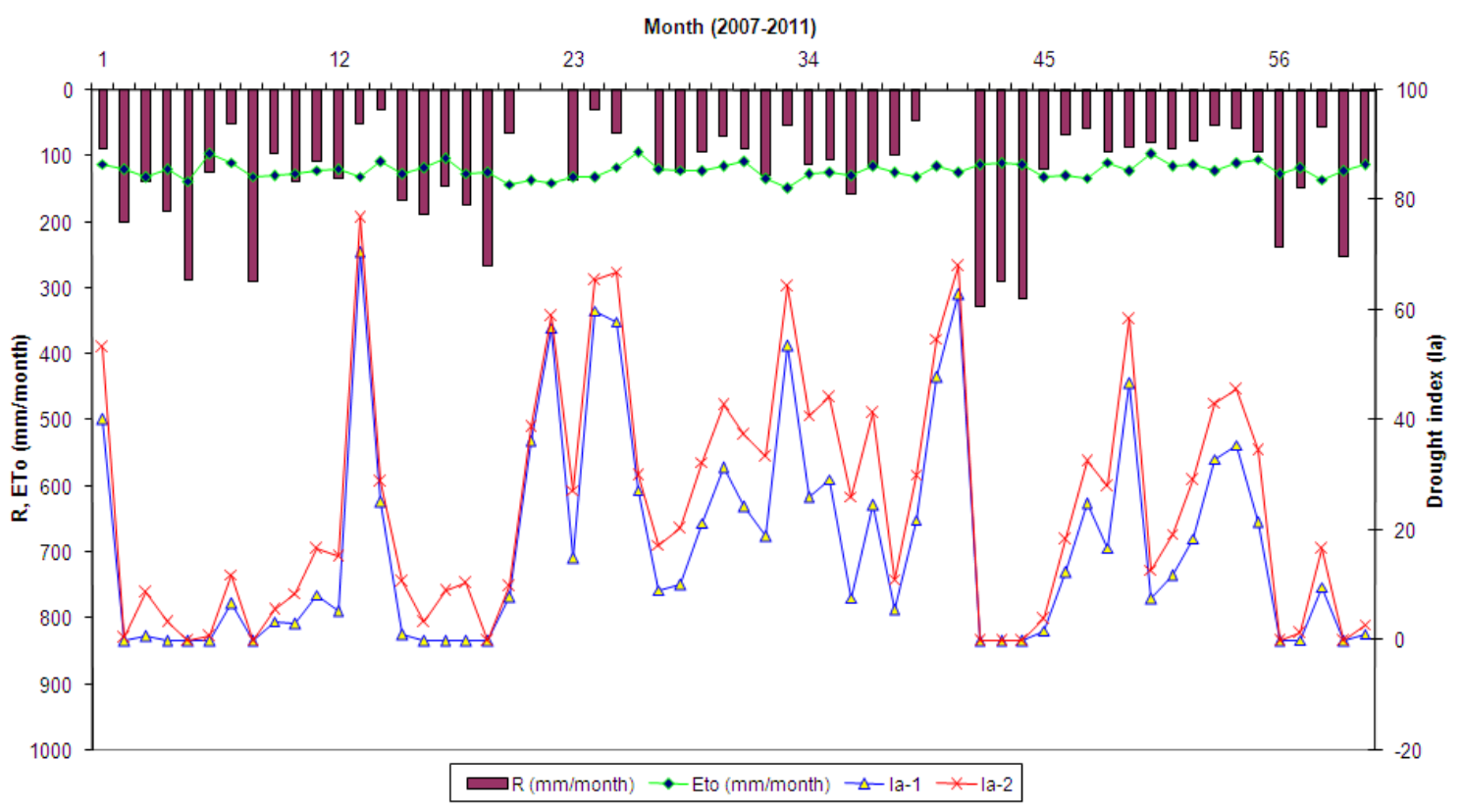

Fig. 4. Correlation between R and ETo with Ia from 2007 to 2011

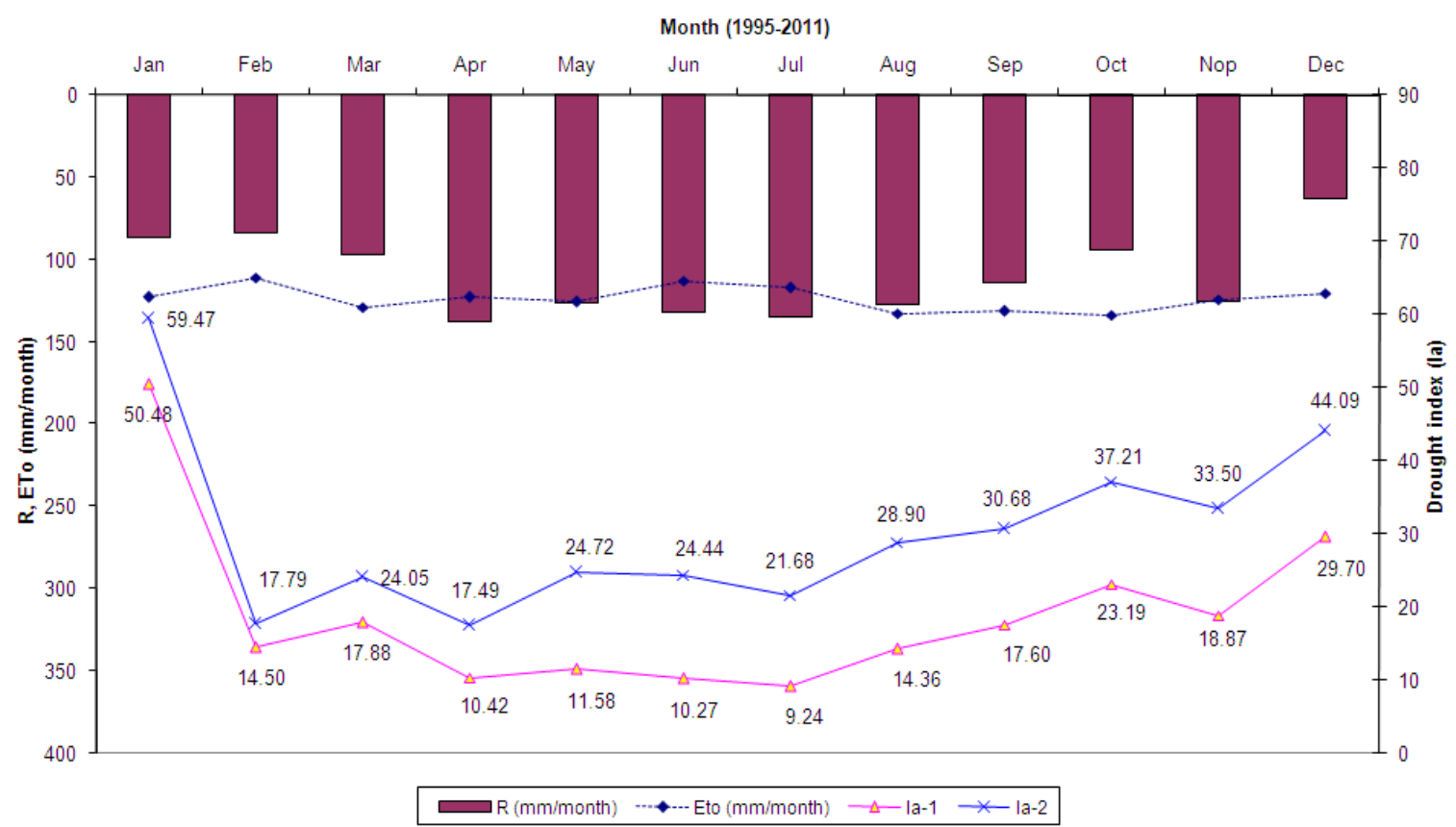

Fig. 5. Correlation between R and ETo with Ia in monthly average from 1995 to 2011

Based on the analysis as in Table 2 and it also presented as in Figure 2 until 4, it could be explained that the value of potential evapotranspiration which was almost the same along the year, so the drought index (Ia) was very influenced by the value of rainfall (R). In 1995, 1996, and 2007, the value of drought index was relatively low that the other years. It was due to the rainfall which was relatively higher than evapotranspiration. The highest value of drought index has happened in 2005 such as 33.65 and the lowest has occurred in 1995. It indicated that in 2005 there was happened the drought for a long time. However, in 1995 there was almost not happened drought except in December on a small scale.

The correlation among rainfall, evapotranspiration, and drought index as in Figure 5 indicated that the average of drought index with the small scale has occurred on February until August, a medium scale has occurred between September until December, and the big scale has occurred on December. The analysis result of drought index before climate change (Ia-1) and after climate change (Ia-2) indicated that there was the increasing of drought index between 15\% until $60 \%$ after climate change which medium drought index has happened on February until September and the big drought index has occurred on January, October until December. By being 
the climate change, there was happened the drought or dry season for a long enough time and there was no drought in small scale.

\section{CONCLUSIONS}

Based on the measurement data of monthly rainfall and climate (hydro-climatology) in the period of 1980 until 2011 which the water balance was analyzed by using the method of Mock-Wyn-UB, it could be concluded that there was happened the increasing of drought index value of $15 \%$ until $60 \%$ after climate change and it indicated that there was happened dry season in long enough time.

\section{AKNOWLEDGEMENT}

The authors thanks very much to the River Regional Department Office of Sulawesi III in Palu, Central Sulawesi, Indonesia and also Watershed Management Department Office of PaluPoso in Center Sulawesi Province which prepared the hydro-climatology data and land use map for supporting this research.

\section{REFERENCES}

[1] Indarto, Hydrology Basic Theory and Application Examples Hydrological Models, 1st ed., Jakarta, Indonesia, BumiAksara, 2010.

[2] Hoybye, Rosbjerg, "Effect of Input and Parameter Uncertainties in Rainfall-Run off Simulations", J.Hydrologic Engineering, vol.4, no.3, pp. 214-224, 1999.

[3] Dzhamalov, R.G.Frolova, N.I. Kireeva, M.B. Safronova T.I., "Climate-Induced Changes in Ground water Runoff in Don Basin", Journal of Water Resource, vol. 17, no. 5, pp.733-742, 2010.

[4] Andawayanti, Ussy, Lily Montarcih, "Climate Change Effect on Water Balance at Pekalen Watershed East Java Province of Indonesia”, International Journal of Academic Research, vol.3, no. 2, pp. 813-816, 2011.

[5] Shotiros Protong, Paul A. Carling, and Julian Leyland, Engineering Journal, vol.22, no. 1, pp. 243-255, 2018.

[6] Simulations, Journal of Hydrologic Engineering, vol.4, no. 3, pp. 214-224, 1999.

[7] Suhardjono, Lily Montarcih, Soemarno, EkoNurhayati, "Verification of Discharge Model Based on Water Balance in Cobanrondo Watershed of East Java", International Journal of Academic Research, vol.2, no. 6, pp. 315-317, 2010.

[8] Sutapa, I.W., Moh. Bisri, Rispiningtati, Lily Montarcih, "Modeling Discharge of the Bangga Watershed Under Climate Change", J. Applied Mechanics and Materials. Vol.776, pp.133-138, 2015.

[9] Vangelis Harris, Spilliotis Mike, Tsakiris, George, "Drought Severity Assessment Based on Bivariate Probability Analysis", J. Water Resource Manage, vol.25, no. 1, pp. 357-371, 2011.

[10] Nasution, Djazim Syaifullah, "Spatial Analysis of the North Coast Regional Drought Index (North Coast) in West Java", Journal Air Indonesia (JAI), vol.1, no. 2, pp. 235-243, 2005.

[11] Sutapa, I.W., "Calibration Parameters Model MockWyn-UB for Calculating Mean Discharge of Bangga River", Asian Academic Research Journal of Multidisciplinary, vol. 1, no. 22, pp 411-421, 2014.

[12] Sutapa, IW.,Moh. Bisri, Rispiningtati, Lily Montarcih. Effect of Climate Change on Water Availability of Bangga River, Central Sulawesi of Indonesia, J.Basic Appl. Sci. Res., vol.3, no. 2, pp.1051-1058, 2013.

[13] Sutapa, I.W., Application Model Mann-Kendall and Sen'S(Make Sens) for Detecting Climate Change, Infrast.J. Civil Eng. Univ.Tadulako, vol.4, no.1, pp.31-40, 2014.

[14] Sutapa, I.W., Long-Term Trend Climatology in Sigi, Central Sulawesiprovince, Seminar on Civil Eng. Narotama of University, 2015 February 28; Surabaya, Indonesia, p.267-277.

[15] Sutapa, I.W., Application of Non-Parametric Test to Detect Trend Rainfall in Watershed Palu, Central Sulawesi, Indonesia, Int. J. Hydrol. Sci. Tech., vol. 6, no. 3, pp.238-253, 2016.

[16] Sutapa, IW., The Influence of Climate Change on Discharge Modeling, Post Graduate Dissertation of Brawijaya University, Malang, East Java, Indonesia, 2013

[17] Sutapa, IW., Moh. Bisri, Rispiningtati, Lily Montarcih, Model of MockWyn-UB for Assessing Water Availability due to the Effect of Climate Change, Proceedings of the International Seminar of Indonesian Hydraulic Engineering Experts Association, Yogyakarta, Indonesia, 8 September 2013.

[18] Sutapa, IW., "Effect of the Climate Change on Groundwater Recharging in Bangga Watershed, Central Sulawesi, Indonesia", Journal Environ. Eng. Res., vol. 22, no. 1, pp. 87-94, 2017.

[19] Aksu. Hakan, Kuşçu. Savaş, and Şimşek. Osman, Trend Analysis of Hydrometeorological Parameters in Climate Regions of Turkey, Jurnal BALWOIS, 2010, Vol.25, pp. 1-7.

[20] Allen G. Richard, Crop Evapotranspiration-Guidelines for Computing Crop Water Requirement-FAO Irrigation and Drainage Paper No. 56, Food Agriculture Organization of the United Nation, Roma, 1998, p. chapter 2.

[21] Kendall, M.G., Mann Kendall Analysis for the Fort http://dubaobien.vn/dhkhtn/stores/files/0907_Tailieu_CuaLo/TLTK/MANN-KENDALL\%20ANALYSIS.pdf,2005, December 4, 2010

[22] Nasution and Djazim Syaifullah, Spatial analysis of the North Coast regional drought index (North Coast) in West Java, Jurnal Air Indonesia (JAI), 2005, vol.1, no. 2, pp. 235-243.

[23] Timo Salmi, Anu Maatta, Pia Anttila, Tuija Ruoho Airola, and Toni Amnell, Detecting Trends of Annual Values of Atmospheric Pollutants by the Mann-Kendall Test and Sen's Slope Estimates, Finnish Meteorological Institute, Helsinki, 2002.

[24] Onoz B, Bayazit M. The power of the statistical test for trend detection.Turkish J. Eng. Env. Sci. 2002, vol. 27, pp. 247-251. 